\title{
Stridor in the Pediatric Patient
}

Sarah Komline, MD; Garima Sarda, MD; Joel Clingenpeel, MD, MPH, MS.MEdL

\section{The distinct features of the pediatric airway make respiratory failure an important concern independent of the underlying cause.}

\section{Cases}

\section{Case 1}

It's a busy shift on an unusually chilly and rainy July night. Emergency medical services (EMS) brings in a 9-month-old boy who woke up with a "squeaking" noise. His parents reported that he has had a fever, cough, rhinorrhea, and difficulty breathing for the past 2 days; however, they did not hear the noisy breathing until the night of presentation. When the patient is examined, it is noted that he has inspiratory stridor at rest, moderate subcostal retractions, and an occasional deep cough. Upper airway transmitted noises were present, but otherwise the patient had clear lungs.

The patient's vital signs at presentation were: blood pressure (BP), 85/55 mm Hg; heart rate (HR), 163 beats/min; respiratory rate (RR), 55 breaths/min; and temperature (T), $101.8^{\circ} \mathrm{F}$. Oxygen saturation was $90 \%$ on room air. The patient's mother wants to know how the respiratory distress will be fixed and is inquiring if they will have to stay in the hospital overnight.

\section{Case 2}

As work begins on the child described above, EMS brings in a 3-year-old girl who appears to be in moderate-severe respiratory distress. Her parents report that she started to drool earlier in the day followed by coughing and occasional gagging. Her parents relay that they thought the symptoms were because of post-nasal drip due to her cold, but the respiratory distress seems to be getting worse, and she now has very noisy breathing and is reluctant to lay down. Upon examination, both inspiratory and expiratory stridor is heard, and it is noted that moderate subcostal retractions are present when the patient is supine.

The patient's vital signs at presentation were: BP, 89/58 mm Hg; HR, 144 beats/min; RR, 52 breaths $/ \mathrm{min}$; and $\mathrm{T}, 99.5^{\circ} \mathrm{F}$. Oxygen saturation was $88 \%$ on room air. The nursing staff asked what to do next and why the 2 stridor cases are being managed so differently.

\section{Stridor}

Stridor is a high-pitched, harsh sound heard during respiration, predominantly during inspiration, as a result of turbulent air passage..$^{1-3}$ Stridor is not a diagnosis in itself, but rather a sign of underlying acute or chronic etiology, which needs to be classified based

Dr Komline is an instructor of pediatrics, Eastern Virginia Medical School, Norfolk. Dr Sarda is a pediatric emergency medicine fellow, Eastern Virginia Medical School, Norfolk. Dr Clingenpeel is the fellowship director of pediatric emergency medicine; and associate professor of pediatrics, Eastern Virginia Medical School/Children's Hospital of The King's Daughters, Norfolk.

Authors' Disclosure Statement: The authors report no actual or potential conflict of interest in relation to this article. 
on elicited history and examination. ${ }^{1,4}$ While the etiology of obstruction may be infectious, congenital, mechanical, or traumatic, the distinct features of the pediatric airway make respiratory failure an important concern independent of the underlying cause.

\section{Anatomy}

There are several anatomical differences unique to the pediatric airway that make children, especially infants under 1 year of age, more susceptible to airway obstruction. ${ }^{5,6}$ The pediatric larynx is more anterior and superior and less fibrous than adults, thereby more compliant. ${ }^{6,7}$ The pediatric epiglottis is longer, omega-shaped, and softer, and the tongue is larger in comparison to the size of the oral cavity, resulting in obstructed airflow. ${ }^{6,7} \mathrm{Chil}-$ dren also have a larger and more prominent occiput, causing mechanical obstruction by flexion of the neck when supine. ${ }^{6}$ While the cricoid cartilage was previously believed to be the narrowest portion of the airway, more recent measurement techniques have challenged this and shown that the glottic and subglottic areas may be narrower in children. ${ }^{7,8}$

Worsening obstruction resulting in a decreased airway radius leads to increased turbulence to air flow, which is explained by Poiseuille's law. ${ }^{1,2,7}$ The resistance to airflow becomes inversely related to the fourth power of the radius, so even a small change in an already narrow pediatric airway can make a huge difference. ${ }^{5}$ In practice, this means $1 \mathrm{~mm}$ of mucosal edema will decrease the cross-sectional area of the airway by $75 \%$ and increase the resistance of airflow by a magnitude of 16 to 32 times depending on the level of turbulence. ${ }^{7}$

The airway can be divided into extrathoracic and intrathoracic regions, separated by the vocal cords. ${ }^{13}$ Inspiratory stridor is typically due to extrathoracic obstruction and expiratory stridor is due to intrathoracic obstruction below the level of the cords. Biphasic stridor, however, may indicate a fixed obstruction at the level of the cords. ${ }^{2,3,5,9}$

\section{Acute Differentials}

The pediatric patient is at high risk for respiratory decompensation when the upper airway is acutely compromised. The history, physical examination, and phases of stridor can help determine the underlying diagnosis and definitive treatment plan.

\section{Acute Croup}

Acute croup (laryngotracheitis) is a clinical diagnosis based on acute onset of barky cough and inspiratory stridor. ${ }^{1}$ It is usually secondary to an infection, most commonly viral (parainfluenza virus), resulting in edema and increased secretions of the subglottic mucosa. ${ }^{7}$ The onset is typically preceded by upper respiratory illness (URI) symptoms, and is often worse at night or after waking from a nap. ${ }^{1}$ The peak incidence is between ages 6 months to 3 years. While croup is a clinical diagnosis, an anteroposterior X-ray will often show a steeple sign. ${ }^{7,10}$

\section{Spasmodic Croup}

Spasmodic croup is an atypical presentation usually seen in children 8 years or older without a preceding URI. Patients will wake up overnight with a harsh brass-like cough, stridor, and hoarse voice. The etiology is unclear, but often these patients have a history of atopy and respond in part to treatment with antihistamines.

\section{Foreign Body Aspiration}

All that is acutely stridulous is not croup. Stridor from foreign body aspiration is sudden in onset and children do not always present with a history suggestive of foreign body 
aspiration. Diagnosis requires a high index of suspicion because the event is often unwitnessed and typical patients are pre-verbal. The physical examination may reveal diminished air entry along with stridor. Diagnosis is made by obtaining an X-ray (lateral neck [Figure 1], lateral decubitus, or inspiratory/expiratory chest X-ray) or by bronchoscopy which is both diagnostic and therapeutic. ${ }^{7,10}$ Remember to always think of foreign body aspiration in children with acute stridor who have neither fever nor antecedent URI symptoms.

\section{Bacterial Tracheitis}

Bacterial tracheitis should be suspected in toxic appearing children who present with respiratory distress and stridor but who have a poor response to nebulized epinephrine. It is typically seen in toddlers and school-aged children, and like a viral tracheitis, presentation can be preceded by either URI or fever. ${ }^{10}$ Stridor is caused by subglottic edema and mucopurulent secretions in the airway. ${ }^{7,10}$ Infection is most commonly by $S$. aureus but initial antibiotic choice should be broad spectrum, and include a third-generation cephalosporin or beta-lactamase resistant penicillin.

\section{Epiglottitis}

Epiglottitis is edema of the epiglottis, most commonly secondary to bacterial infection. The epidemiology of epiglottitis has changed dramatically since widespread immunization for $\mathrm{H}$. influenza with a significantly decreased incidence and change in the average age of presentation to 14.6 years (previously 5.6 years). ${ }^{7}$ The clinical course begins with sore throat, dysphonia, refusal to eat and progressive difficulty handling secretions with eventual drooling, stridor, tripoding, and toxic appearance. Epiglottitis can be differentiated from croup and bacterial tracheitis because presentation typically lacks a cough. ${ }^{7,10,11}$ Diagnosis is made either by direct visualization of the epiglottis or a lateral neck X-ray showing a 'thumb print' sign (Figure 2). ${ }^{12}$ Emergency department treatment is similar to the management of the child with a partial foreign body occlusion and focuses on maintaining the airway and minimizing anything that agitates the patient. Intravenous (IV) antibiotic coverage is similar to bacterial tracheitis (third-generation cephalosporin or a beta-lactamase resistant penicillin).

\section{Retropharyngeal Abscess}

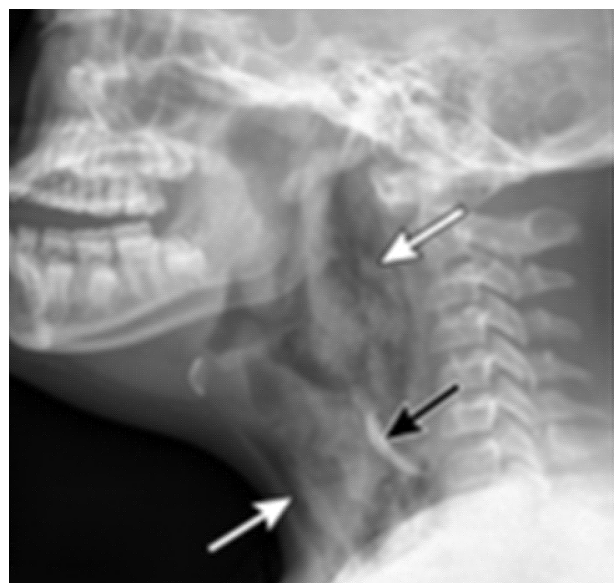

Figure 1. Fishbone (black arrow) in trachea causing tracheoesophageal fistula and subcutaneous air (white arrows).

Reprinted with permission from Darras KE, Roston AT, Yewchuk LK. Imaging acute airway obstruction in infants and children. RadioGraphics. 2015;35:2064-2079.

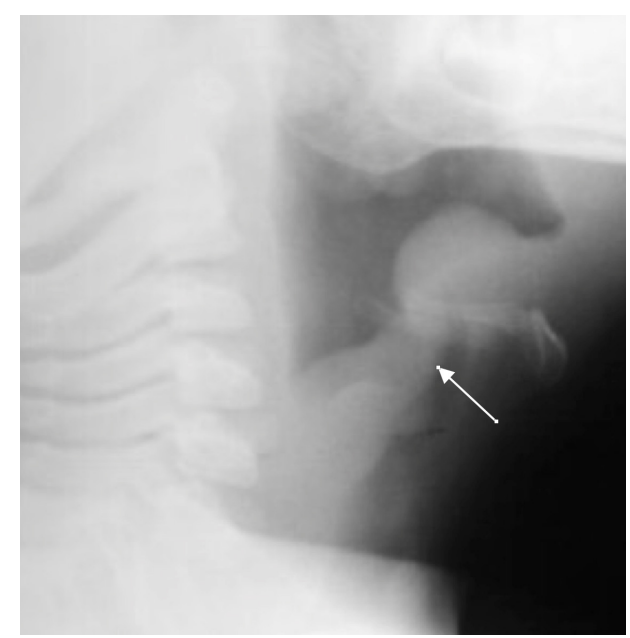

Figure 2. Thumbprint sign is indicative of epiglottitis (white arrow). On lateral neck X-ray, the epiglottis appears more radiopaque secondary to edema and enlargement.

Reprinted with permission from Loren G. Yamamoto, MD, $\mathrm{MPH} .{ }^{23}$

The most common chief complaint of retropharyngeal abscess (RPA) is neck pain (38\%) with fever. As such, it can clinically be mistaken for meningitis on initial presentation. Retropharyngeal abscess will present rarely with either stridor or associated respiratory distress, and it can also mimic croup on initial pre- 
sentation. Physical examination findings which differentiate this entity include limited or painful neck extension (45\%), torticollis (36.5\%), and to a lesser extent limitation of neck flexion $(12.5 \%) .{ }^{13}$ The median age at diagnosis is 36 months with $75 \%$ of patients less than 5 years. Typical presentation is insidious with fever and URI symptoms preceding onset. Diagnosis can be made with a lateral neck X-ray showing widening of the prevertebral space (Figure 3), but the gold standard diagnostic study is a computed tomography with contrast. ${ }^{14}$ Management is IV antibiotics covering aerobic and anaerobic bacteria (eg, ampicillin-sulbactam) \pm surgical intervention.

\section{Caustic Ingestion}

Caustic ingestion is most commonly accidental and seen in children aged 12 months to 2 years. However, with recent fads, such as the "Tide Pod challenge" teenagers are also at risk. Airway compromise and stridor are secondary to mucosal injury and edema. Oral injury is not always a useful marker for significant distal injury. A complete evaluation of the upper airway and digestive tract within 48 hours after known/suspected caustic ingestion is recommended to assess full extent of damage. ${ }^{15}$

\section{Chronic Differentials Laryngomalacia}

Laryngomalacia is a congenital weakness of laryngeal tissues, and it

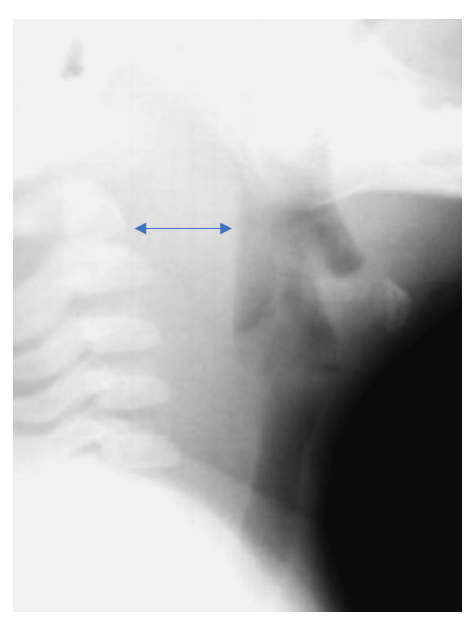

Figure 3. Potential for severe airway obstruction from pediatric retropharyngeal abscess. The prevertebral space (double-headed arrow) is wider than the width of the vertebral body. Reprinted with permission from Loren $\mathrm{G}$. Yamamoto, MD, MPH. ${ }^{23}$ is the most common cause of both chronic stridor and neonatal stridor. It is characterized by progressive worsening of symptoms with crying/feeding and supine positioning. Diagnosis is made by bronchoscopy and management is conservative unless there are life threatening apneic or cyanotic events. ${ }^{7}$

\section{Rings/Slings}

There are many anatomic structures with the potential to cause extrinsic airway compression which present with stridor. This type of stridor is often biphasic. Examples include innominate artery compression, double aortic arch, aberrant subclavian artery, and pulmonary artery sling. ${ }^{7}$

Stridor presenting in children with a history of prematurity or prolonged intubation should raise concern for subglottic and tracheal stenosis. ${ }^{16}$

\section{Evaluation}

Regardless of the etiology of stridor, efforts should be made to keep the patient calm (ie, allow the parent to keep holding a young child, limit any examination not absolutely necessary). Much of the examination can be completed from a distance without disturbing the child.$^{17}$ Observation of the inspiratory:expiratory (I:E) ratio can localize the level of airway obstruction. For example, an I:E ratio weighted toward a longer inspiration indicates an extrathoracic airway obstruction. Whereas an I:E with a prolonged expiratory phase is consistent with intrathoracic obstruction (eg, terminal bronchial obstruction). ${ }^{17}$

Another way to localize the level of obstruction is to look for changes in the voice; patients who present with a change in their voice have a subglottic partial obstruction such as croup. However, patients with a muffled voice or drooling have a supraglottic obstruction such as epiglottitis or RPA. ${ }^{17}$ 


\section{Management}

Management of stridor focuses on reducing airway obstruction, which is usually secondary to edema in the acute setting.

\section{Viral Laryngotracheitis}

Oral steroids are the mainstay of treatment. Research has shown dexamethasone is preferred over prednisolone. ${ }^{18-20}$ Steroids are not only useful in moderate to severe laryngotracheitis but also have a therapeutic role in children with mild laryngotracheitis. ${ }^{18}$ In hospital settings the parenteral formulation of dexamethasone can be safely given orally with good effect. There is no therapeutic advantage in acute laryngotracheitis to giving dexamethasone via either the IV or intramuscular route vs oral. ${ }^{21}$ In the outpatient setting, decadron tablets can be crushed and mixed in with a young child's favorite soft food (eg, mashed potatoes or apple sauce). The authors recommend this strategy in lieu of prescribing dexamethasone suspension as its dilute concentration $(1 \mathrm{mg} / 10$ $\mathrm{ml}$ ) results in a need for a child to receive a relatively large volume of a distasteful liquid. There is a wide therapeutic range of dexamethasone with studies documenting efficacy for laryngotracheitis in doses ranging from $0.15 \mathrm{mg} / \mathrm{kg}$ to 0.6 $\mathrm{mg} / \mathrm{kg}$. To date there are no large studies which demonstrate routine therapeutic utility of subsequent doses of dexamethasone. Nebulized budesonide $(2.5 \mathrm{mg})$ can be given if oral steroids are not tolerated, however it is significantly more expensive.

Racemic epinephrine is the agent of choice for rapid onset of action in children who demonstrate stridor at rest. It causes vasoconstriction in the laryngeal mucosa, promotes bronchial smooth muscle relaxation, and thinning of bronchial secretions. It offers short-term relief of symptoms until steroids start to work. There is no rebound effect or worsening of symptoms once the epinephrine wears off, but children who receive this drug should be observed in the ED for a period of time (2-3 hours is standard of care in many hospitals) for return of symptoms. ${ }^{10,22}$ Patients who are persistently symptomatic 4 hours after administration of steroids or who require repeat doses of racemic epinephrine should be admitted for observation. ${ }^{10}$

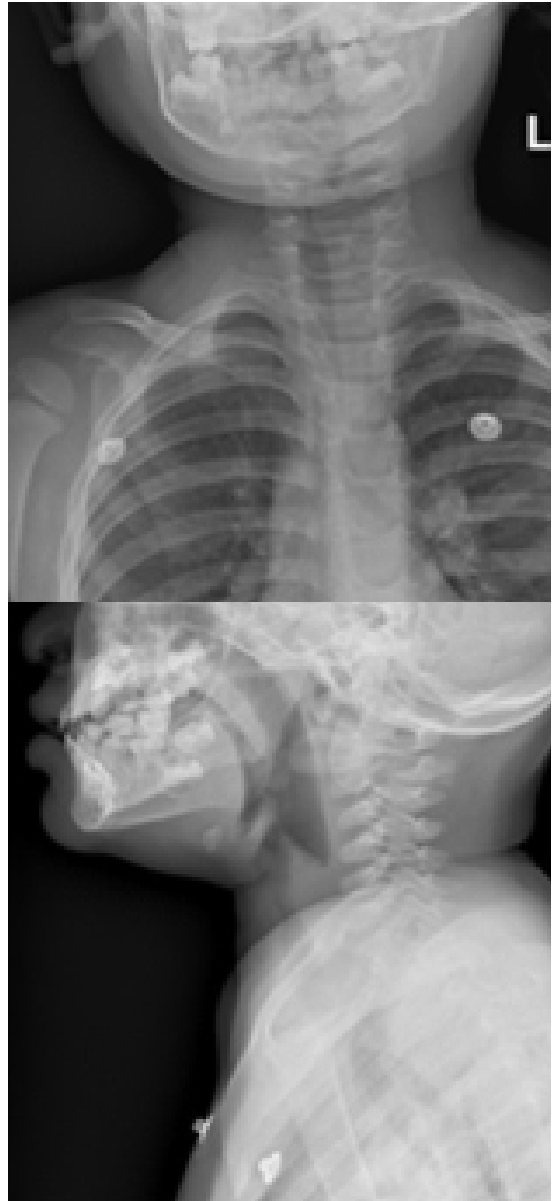

Figure 4. Subglottic narrowing is visible on an anteroposterior view of a neck X-ray in a patient with croup.

There are no contraindications to adjuvant treatments, such as antipyretics and non-sedating analgesics. Clinicians should maintain a high index of suspicion for anatomic airway anomalies that may need further evaluation/direct visualization in pediatric patients who present with repeated episodes of croup. ${ }^{10}$

\section{Case Conclusions}

\section{Case 1}

The 9-month-old boy with stridor was noted to have increased stridor while fussy, but even at rest some inspiratory stridor was present. A barky cough was noted in the examination room. The patient was placed on a monitor and a nebulized racemic epineph- 
rine treatment was started. A single dose of oral dexamethasone was given shortly after presentation to the ED. Since the patient had inspiratory stridor at rest with associated tachypnea and hypoxia on initial presentation, a neck X-ray was obtained (Figure 4). Subglottic narrowing was identified on the imaging, but both the epiglottis and the prevertebral space were normal in appearance and no foreign bodies were visualized. The inspiratory stridor at rest, tachypnea, and mild hypoxia all improved after treatment, the patient was observed for 2 hours in the ED without recurrence of respiratory distress and was able to be discharged home with a diagnosis of acute croup.

\section{Case 2}

The 3-year-old girl was noted to be in significant positional respiratory distress, so the physician asked her parents to keep her calm in her position of comfort. She was calmly and quickly placed on a monitor with age-appropriate distraction techniques in place and advanced airway equipment at the bedside. A portable chest X-ray was obtained and revealed a coin was partially obstructing the trachea. Care was taken in the ED to avoid all interventions such as IV access that might upset the child so as not to inadvertently convert this partial airway obstruction to a complete obstruction. The otolaryngology team was called urgently, and the patient was transported to the operating room for foreign body removal in a controlled environment.

\section{References}

1. Bjornson CL, Johnson DW. Croup. Lancet. 2008;371(9609):329-339. doi:10.1016/S0140-6736(08)60170-1.

2. Escobar ML, Needleman J. Stridor. Pediatr Rev. 2015;36(3):135-137. doi:10.1542/pir.36-3-135.

3. Pfleger A, Eber E. Assessment and causes of stridor. Paediatr Respir Rev. 2016;18:64-72. doi:10.1016/j. prrv.2015.10.003.

4. Boudewyns A, Claes J, Van de Heyning P. Clinical practice: an approach to stridor in infants and children. Eur J Pediatr. 2010;169(2):135-141. doi:10.1007/s00431-009-1044-7.

5. Kelley PB, Simon JE. Racemic epinephrine use in croup and disposition. Am J Emerg Med. 1992;10(3):181-183.

6. Mandal A, Kabra SK, Lodha R. Upper airway obstruction in children. Indian J Pediatr. 2015;82(8):737-744.

7. Marchese A, Langhan ML. Management of airway obstruction and stridor in pediatric patients. Pediatr Emerg Med Pract. 2017;14(11):1-24.

8. Wani TM, Bissonnette B, Rafiq Malik M, et al. Age-based analysis of pediatric upper airway dimensions using computed tomography imaging. Pediatr Pulmonol. 2016;51(3):267-271. doi:10.1002/ppul.23232.

9. Donaldson D, Poleski D, Knipple E, et al. Intramuscular versus oral dexamethasone for the treatment of moderateto-severe croup: a randomized, double-blind trial. Acad Emerg Med. 2003;10(1):16-21.

10. Boudewyns A, Claes J, Van de Heyning P. Clinical practice: an approach to stridor in infants and children. Eur J Pediatr. 2010;169(2):135-141. doi:10.1007/s00431-009-1044-7.

11. Tibballs J, Watson T. Symptoms and signs differentiating croup and epiglottitis. J Paediatr Child Health. 2011;47(3):77-82. doi:10.1111/j.1440-1754.2010.01892.x.

12. Sobol SE, Zapata S. Epiglottitis and croup. Otolaryngol Clin North Am. 2008;41(3):551-566. doi:10.1016/j. otc.2008.01.012.

13. Craig FW, Schunk JE. Retropharyngeal abscess in children: clinical presentation, utility of imaging, and current management. Pediatrics. 2003;111(6 Pt 1):1394-1398.

14. Roberson DW. Pediatric retropharyngeal abscesses. Clin Pediatr Emerg Med. 2004;5(1):37-40.

15. Riffat F, Cheng A. Pediatric caustic ingestion: 50 consecutive cases and a review of the literature. Dis Esophagus. 2009;22(1):89-94. doi:10.1111/j.1442-2050.2008.00867.x.

16. Daniel SJ. The upper airway: congenital malformations. Paediatr Respir Revi. 2006;7 Suppl 1:S260-S263.

17. Arutyunyan H, Spangler M. Pediatric upper airway obstruction. Peds RAP Web site. https://www.hippoed.com/ peds/rap/episode/pedsrapfebruary/pediatricupper. Published February 2018. Accessed August 31, 2018.

18. Bjornson CL, Klassen TP, Williamson J, et al; Pediatric Emergency Research Canada Network. A randomized trial of a single dose of oral dexamethasone for mild croup. N Engl J Med. 2004;351(13):1306-1313.

19. Fifoot AA, Ting JY. Comparison between single-dose oral prednisolone and oral dexamethasone in the treatment of croup: a randomized, double-blinded clinical trial. Emerg Med Australas. 2007;19(1):51-58.

20. Sparrow A, Geelhoed G. Prednisolone versus dexamethasone in croup: a randomised equivalence trial. Arch Dis Child. 2005;91(7):580-583.

21. Donaldson D, Poleski D, Knipple E, et al. Intramuscular versus oral dexamethasone for the treatment of moderateto-severe croup: a randomized, double-blind trial. Acad Emerg Med. 2003;10(1):16-21.

22. Kelley PB, Simon JE. Racemic epinephrine use in croup and disposition. Am J Emerg Med. 1992;10(3):181-183.

23. Yamamoto LG. Test your skill in reading pediatric lateral necks. University of Hawaii Web site. https://www. hawaii.edu/medicine/pediatrics/pemxray/v2c20.html. Accessed September 13, 2018. 